\title{
Bioequivalence Study of Donepezil Hydrochloride Tablets in Healthy Male Volunteers
}

\author{
Noppamas Rojanasthien, Siriluk Aunmuang, Nutthiya Hanprasertpong, \\ Sukit Roongapinun, and Supanimit Teekachunhatean \\ Department of Pharmacology, Faculty of Medicine, Chiang Mai University, Chiang Mai 50200, Thailand \\ Correspondence should be addressed to Supanimit Teekachunhatean, steekach@med.cmu.ac.th
}

Received 15 September 2012; Accepted 3 October 2012

Academic Editors: F. J. Miranda and T. B. Vree

Copyright (c) 2012 Noppamas Rojanasthien et al. This is an open access article distributed under the Creative Commons Attribution License, which permits unrestricted use, distribution, and reproduction in any medium, provided the original work is properly cited.

\begin{abstract}
The objective of this study was to investigate the bioequivalence of two formulations of $5 \mathrm{mg}$ donepezil HCL tablets: Tonizep as the test and Aricept as the reference. The two products were administered as a single oral dose according to a randomized two-phase crossover with a 3-week washout period in 20 healthy Thai Male volunteers. After drug administration, serial blood samples were collected over a period of 216 hours. Plasma donepezil concentrations were measured by high performance liquid chromatography with UV detection. Pharmacokinetic parameters were analyzed based on noncompartmental analysis. The logarithmically transformed data of $\mathrm{AUC}_{0-\infty}$ and $C_{\max }$ were analyzed for $90 \%$ confidence intervals (CI) using ANOVA. The mean $(90 \% \mathrm{CI})$ values for the ratio of $\mathrm{AUC}_{0-\infty}$ and $C_{\max }$ values of the test product over those of the reference product were 1.08 (1.021.14 ) and $1.08(0.99-1.17)$, respectively (within the bioequivalence range of $0.8-1.25)$. The median $T_{\max }$ for the test product was similar to that of the reference product $(2.0 \mathrm{hr})$, and the $90 \% \mathrm{CI}$ for the $T_{\max }$ difference between the two preparations was -0.19 to $0.29 \mathrm{hr}$ and within the bioequivalence range of $\pm 20 \%$ of the $T_{\max }$ of the reference formulation. Our study demonstrated the bioequivalence of the two preparations.
\end{abstract}

\section{Introduction}

Donepezil is a reversible inhibitor of the enzyme acetylcholinesterase (AchE) approved for use in Alzheimer's disease $[1,2]$. The pathogenesis of Alzheimer's disease attributed some of them to a deficiency of cholinergic neurotransmission. Therefore, AChE inhibitors, which prevent the hydrolysis of acetylcholine, may exert their therapeutic effect by enhancing cholinergic function. The first AChE inhibitor (tacrine) has been used, however, associated with a high incidence of gastrointestinal (GI) side effects and hepatotoxicity [3]. Donepezil is a potent and more selective AChE inhibitor in the central nervous system with little effect on peripheral tissue, therefore, has a lower incidence of GI and cardiovascular adverse effects [1]. The drug produces modest improvements in cognitive scores and has a long halflife allowing once daily dosing [4].

Donepezil is slowly absorbed from the GI tract. Its maximal plasma concentrations $\left(C_{\max }\right)$ were reached within 3-4 hours. Its relative oral bioavailability is $100 \%$ and food did not affect its absorption [5-10]. The average $C_{\max }$ after $5 \mathrm{mg}$ dose ranged from 7.7 to $10.1 \mathrm{ng} / \mathrm{mL}$ [5-7]. Pharmacokinetics are linear over a dose range of $1-10 \mathrm{mg}$ given once daily [8-10]. Neither food nor time of administration influences the rate and extent of its absorption. It is approximately $96 \%$ bound to plasma proteins. The drug is both excreted by the kidney and extensively metabolized by the liver CYP 450 (2D6 and 3A4) as well as glucuronidation [1]. The elimination half-life is about $50-70$ hours and the mean apparent plasma clearance $(\mathrm{Cl} / \mathrm{F})$ is $0.13 \mathrm{~L} / \mathrm{hr} / \mathrm{kg}$ [5-10]. Following multiple doses, it accumulates in plasma by $4-7-$ fold, and the steady-state is achieved within 3 weeks. The steady-state volume of distribution is $12 \mathrm{~L} / \mathrm{kg}$. The starting dose of donepezil is $5 \mathrm{mg}$ administered once daily in the evening. The higher dose may not provide a significant greater benefit, however, may cause higher incidence of cholinergic adverse events [1]. Because steady state is not achieved until 15 days, treatment with a dose of $10 \mathrm{mg}$ should 
not be given until patients have been on a daily dose of $5 \mathrm{mg}$ for 4-6 weeks. Adverse effects of donepezil included, diarrhea, nausea, vomiting, insomnia, muscle cramp, fatigue and anorexia which were often mild in intensity or transient and resolving during continued medication $[1,3]$. Donepezil is well tolerated in patients with mild hepatic impairment and moderately to severely impaired renal function [5]. Overdose can result in cholinergic crisis requiring atropine as an antidote.

A generic preparation of donepezil has been developed for clinical use with a lower cost. Although the generic and the innovator preparations contain the same active ingredient, they differ from each other by manufacturing processes as well as content of excipients, which affect the rate and extent of absorption of active drug. Therefore, the bioequivalence testing is mandated to confirm the bioavailability between the two preparations in human subjects. The objective was to determine the bioequivalence of two oral formulations of $5 \mathrm{mg}$ donepezil tablets when given as equal dose.

\section{Materials and Methods}

2.1. Subjects. Twenty healthy Thai male subjects aged between 20 and 32 years old and the body mass index within 18-25 participated in this study. Subjects were in good health on the basis of medical history, physical examination, and routine blood test. Subjects with known contraindication or hypersensitivity to donepezil were excluded as well as those with history of drug abuse, heavy alcohol consumption or cigarette smoking. No drug was allowed 1 month before the study period. The study was approved by the Research Ethics Committee of the Chiang Mai University.

2.2. Study Drugs. The reference product of $5 \mathrm{mg}$ donepezil was Aricept manufactured by Eisai Taiwan Inc., Tainan Factory, Tainan Hsien, Taiwan, and imported by Eisai (Thailand) Marketing Co., Ltd. Bangkok, Thailand (lot no. 61A15T, Mfd 04/01/2006, Exp 03/01/2009). The test product was Tonizep manufactured by T. O. Chemicals (1979) Ltd., Bangkok, Thailand (lot no. TNZ 5-01, Mfd 14/03/2006, Exp 03/2009).

2.3. Study Design and Method of Drug Administration. This was a randomized, 2-period crossover study. Each subject was randomly assigned to receive a single oral dose of $5 \mathrm{mg}$ donepezil tablet (either Aricept or Tonizep) with $240 \mathrm{~mL}$ water on the morning after an overnight fast. Subjects remained upright for 4 hours and were fasted 2 hours after drug administration. Water and lunch were served 2 hours and 4 hours after dose, respectively. The washout period between each treatment was 3 weeks. After a washout period, subjects were administered the different brand of donepezil in the same manner. An identical meal and fluid intake were served during the two study periods. Subjects were required to refrain from drinking caffeine containing beverages and alcohol. Blood samples were collected immediately before and at 1, 2, 3, 4, 5, 6, 8, 12, 24, 48, 72, 96, 120, 144, 168, 192 and $216 \mathrm{~h}$ after dose. Within 30 minutes, the blood samples were centrifuged to separate the plasma. The plasma samples were immediately kept at $-20^{\circ} \mathrm{C}$ until assay.

2.4. Determination of Donepezil Concentration in Plasma. The assay was operated using a high performance liquid chromatography (HPLC) with UV detector set at $315 \mathrm{~nm}$. The chromatographic system consisted of a $150 \times$ $4.6 \mathrm{~mm}$ i.d., $5 \mu \mathrm{m}$ C18 reversed-phase analytical column, and a $10 \times 4.6 \mathrm{~mm}$ i.d., $5 \mu \mathrm{m} \mathrm{C18}$ guard column. The mobile phase was a mixture of $27.5 \mathrm{mM} \mathrm{KH} \mathrm{KH}_{4}(\mathrm{pH}$ 4.5)/acetonitrile/2-propanol (500/220/5, v/v/v). The system was isocratic operated with a flow rate of $1.0 \mathrm{~mL} / \mathrm{min}$ and set the temperature at $25^{\circ} \mathrm{C}$. Plasma sample of donepezil and internal standard (IS) ondansetron were prepared by solid-phase extraction (SPE) using C18 SPE cartridges, preconditioned with methanol and water. Thereafter, $0.3 \mathrm{~mL}$ of $100 \mathrm{mM}$ of $\mathrm{KH}_{2} \mathrm{PO}_{4}, 0.3 \mathrm{~mL}$ of $20 \%$ 2-propanol, and $1.25 \mathrm{~mL}$ of $20 \%$ methanol/water were sequentially loaded. The SPE cartridge was vacuumed to dryness and eluted with $1.8 \mathrm{~mL}$ of 2-propanol/acetonitrile/perchloric acid (25/75/30, $\mathrm{mL} / \mathrm{mL} / \mathrm{uL}$ ). The eluent was collected and evaporated to dryness by SpeedVac concentrator. The residue was reconstituted with $30 \mu \mathrm{L}$ mobile phase, and $10 \mu \mathrm{L}$ of the reconstituted sample was injected onto the HPLC system.

\subsection{Statistical Methods and Data Analysis}

2.5.1. Pharmacokinetic Analysis. Maximal plasma concentration $\left(C_{\max }, \mathrm{ng} / \mathrm{mL}\right)$ and time to reach the peak concentration $\left(T_{\max }\right.$, hr) were obtained directly by visual inspection of each subject's plasma concentration-time profile. The area under the plasma concentration-time curve (AUC) from time 0 to infinity $\left(\mathrm{AUC}_{0-\infty}, \mathrm{ng} \cdot \mathrm{h} / \mathrm{mL}\right)$ and half-life $\left(t_{1 / 2}, \mathrm{hr}\right)$ were determined by noncompartmental analysis. The slope of the terminal log-linear portion of the concentration-time profile was determined by least-squares regression analysis and used as the elimination rate constant $\left(K_{e}\right)$. The elimination halflife $\left(t_{1 / 2}\right)$ was calculated as $0.693 / K_{e}$. The $\mathrm{AUC}_{0-t}$ from time zero to the last quantifiable point $(\mathrm{Ct})$ was calculated using the trapezoidal rule, and extrapolated AUC from $\mathrm{Ct}$ to infinity $\left(\mathrm{AUC}_{t-\infty}\right)$ was determined as $\mathrm{C}_{t} / \mathrm{K}_{e}$. Total $\mathrm{AUC}_{0-\infty}$ was the sum of $\mathrm{AUC}_{0-t}+\mathrm{AUC}_{t-\infty}$. The calculation was performed by using the TopFit 2.0, pharmacokinetic data analysis program for PC.

2.5.2. Statistical Analysis. An analysis of variance (ANOVA) was performed to determine the statistical differences of pharmacokinetic parameters $\left(\mathrm{AUC}_{0-\infty}, C_{\max }\right.$, and $T_{\max }$ ) which represented the extent and rate of drug absorption. Statistic analysis of AUC and $C_{\max }$ was performed on the logarithmically (ln) transformed data. The $90 \%$ confidence interval (CI) for the ratio of AUC as well as $C_{\max }$ values of the test preparation over those of the reference product were estimated using the following equation $[11,12]$ :

$$
90 \% \mathrm{CI}=\left(\bar{X}_{T}-\bar{X}_{R}\right) \pm t_{0.1}^{v} \sqrt{\frac{2 s^{2}}{n}} .
$$


$\bar{X}_{T}$ and $\bar{X}_{R}$ are the observed means of the (ln) transformed parameters (either $C_{\max }$ or AUC) for the test product $(T)$ and the references $(R) . S^{2}$ is the error variance obtained from the ANOVA, and $n$ is the number of subjects. $t_{0.1}^{v}$ is the tabulated two-tail $t$ value for $90 \%$ CI. $v$ is the number of degree of freedom of the error mean square.

The antilogarithm of the CI would express the bioequivalence as a ratio of the test product and the reference product (test/reference). The bioequivalence acceptance criteria required that the $90 \% \mathrm{CI}$ for the test/reference ratios of the AUC and $C_{\max }$ fell within the interval of 0.8-1.25.

Regarding analysis of $T_{\max }$, the limits for the bioequivalence range were expressed as untransformed data (absolute differences) and the stipulated bioequivalence range of difference $T_{\max }$ [test - reference] was $\pm 20 \%$ of the $T_{\max }$ of the reference formulation [13].

\section{Results and Discussion}

Twenty healthy Thai male volunteers participated in the study. Their mean values of age, weight, height, and body mass index were $24.6 \pm 3.5 \mathrm{yr}, 62.4 \pm 6.5 \mathrm{~kg}, 1.7 \pm 0.1 \mathrm{~m}$, and $21.8 \pm 1.9 \mathrm{~kg} / \mathrm{m}^{2}$, respectively. All completed the study protocol.

The chromatograms of blank plasma and plasma containing IS and $100.0 \mathrm{ng} / \mathrm{mL}$ donepezil are present in Figure 1. The retention time of IS and donepezil was 3.2 and $4.5 \mathrm{~min}$, respectively. The lower limit of quantification (LLOQ) of donepezil in plasma was $2 \mathrm{ng} / \mathrm{mL}$. Calibration curves of donepezil were linear ranged from 2.0 to $100.0 \mathrm{ng} / \mathrm{mL}$, and linear regression of drug concentration versus peak height ratios (donepezil/IS) gave coefficients of determination $\left(r^{2}\right)$ greater than 0.990 . The average recoveries (\%) of IS and donepezil were 102.38 and $97.39 \%$, respectively.

Oral administrations of $5 \mathrm{mg}$ donepezil hydrochloride tablets were well tolerated. The mean plasma concentrationtime curves of Tonizep and Aricept were comparable (Figure 2). Donepezil pharmacokinetic parameters including $C_{\max }, T_{\max }, \mathrm{AUC}_{0-\infty}$, and $t_{1 / 2}$ between the test and the reference were demonstrated in Table 1 . The median $T_{\max }$ for Tonizep was similar to that of Aricept $(2 \mathrm{hr}$, range $1-3 \mathrm{hr})$, and the mean $(90 \% \mathrm{CI})$ for the $T_{\max }$ difference between the two preparations was $0.05(-0.19$ to 0.29$)$ $\mathrm{hr}$ and within the bioequivalence range of $\pm 0.41 \mathrm{hr}$. The average half-life of donepezil in serum between the test (91.5 hr, range 59.1-164 hr) and the reference $(90.7 \mathrm{hr}$, range $62.5-148 \mathrm{hr}$ ) was similar, however, was longer than expected values reported from a previous study $(50-70 \mathrm{hr})$. The mean values $( \pm \mathrm{SD})$ of the $C_{\max }$ and $\mathrm{AUC}_{0-\infty}$ for Tonizep were not significantly different from those of Aricept (20.42 \pm 4.5 versus $18.93 \pm 3.82 \mathrm{ng} / \mathrm{mL}$ and $1375.01 \pm 369.01$ versus $1277.47 \pm 328.51 \mathrm{ng} \cdot \mathrm{hr} / \mathrm{mL}$ ). Bioequivalence analysis showed that $90 \% \mathrm{CI}$ for the test/reference ratios of $\mathrm{AUC}_{0-\infty}$ and $C_{\max }$ were $1.08(1.02-1.14)$ and $1.08(0.99-1.17)$, respectively (Table 2). The coefficient of variation (\%CV) estimated from $S^{2}$ obtained from the ANOVA after logarithmic transformed for $\mathrm{AUC}_{0-\infty}$ and $C_{\max }$ was $10 \%$ and $15 \%$, respectively. According to the nomograms and tables of Diletti, the power

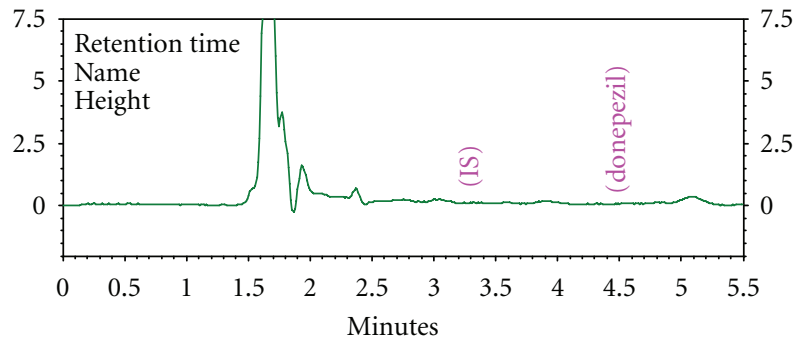

(a)

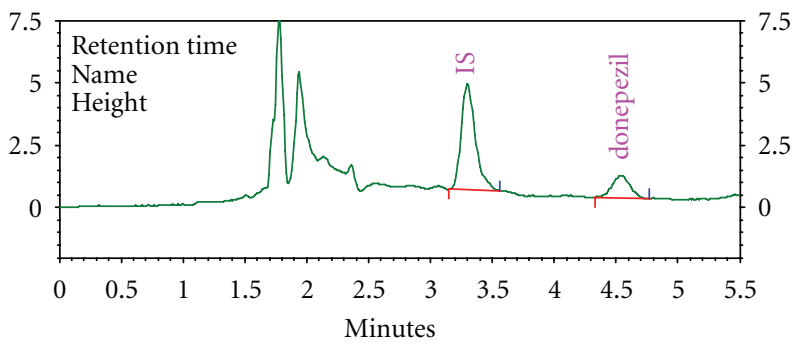

(b)

Figure 1: (a) Chromatogram of donepezil-free plasma. (b) Chromatogram of plasma sample containing internal standard (IS, retention time $=3.294 \mathrm{~min}$ ) and donepezil (retention time $=$ $4.538 \mathrm{~min})$.

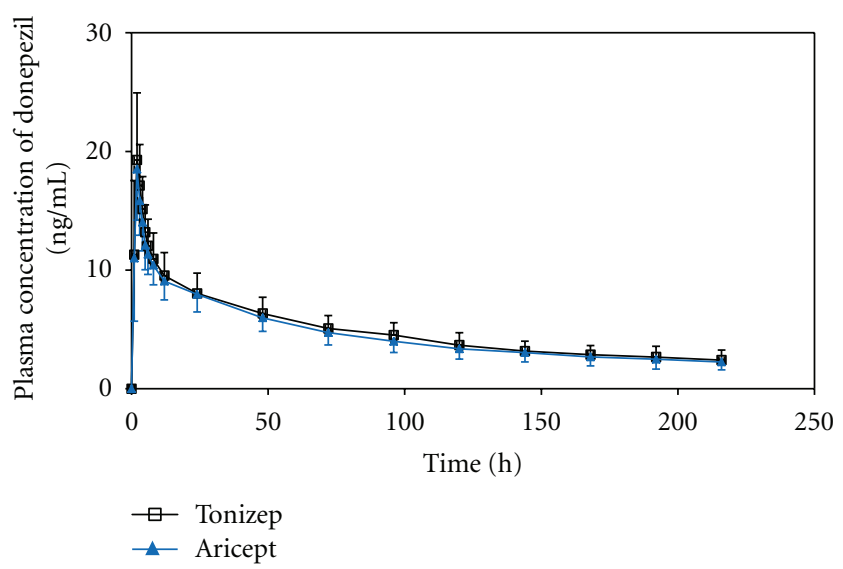

FIGURE 2: Mean plasma concentration-time profiles after oral administration of $5 \mathrm{mg}$ Tonizep and Aricept under fasted condition.

of tests values for AUC and $C_{\max }$ were $>90 \%$ and $80 \%$ for the sample size of 20, respectively. In addition, since the $90 \%$ CI values of $\mathrm{AUC}_{0-\infty}$ and $C_{\max }$ were within the bioequivalence range, our study demonstrated the bioequivalence of the two preparations.

\section{Conclusions}

The study evaluated the bioequivalence of $5 \mathrm{mg}$ oral formulations of donepezil hydrochloride tablet manufactured by the T. O. Chemicals (1979) Ltd., Bangkok, Thailand, (Tonizep) and the innovator Aricept in 20 healthy Thai male subjects. Each subject was given the test and the reference 
TABle 1: Comparison of donepezil pharmacokinetic parameters after single oral dose of $5 \mathrm{mg}$ reference product (Aricept) and test product (Tonizep).

\begin{tabular}{lcc}
\hline $\begin{array}{l}\text { Pharmacokinetic } \\
\text { parameter }\end{array}$ & $\begin{array}{c}\text { Reference product } \\
\text { (Aricept) }\end{array}$ & $\begin{array}{c}\text { Test product } \\
\text { (Tonizep) }\end{array}$ \\
\hline$C_{\max }{ }^{1}(\mathrm{ng} / \mathrm{mL})$ & $18.93 \pm 3.82$ & $20.42 \pm 4.50$ \\
$T_{\max }{ }^{2}(\mathrm{hr})$ & $2.0(1.0-3.0)$ & $2.0(1.0-3.0)$ \\
$\mathrm{AUC}_{0-\infty}{ }^{1}(\mathrm{ng} \cdot \mathrm{hr} / \mathrm{mL})$ & $1277.47 \pm 328.51$ & $1375.01 \pm 369.01$ \\
$t_{1 / 2}{ }^{1}(\mathrm{hr})$ & $90.7 \pm 18.1$ & $91.5 \pm 21.3$ \\
${ }^{1}$ Represented as mean $\pm \mathrm{SD} .{ }^{2}$ Represented as median (range).
\end{tabular}

TABLE 2: The mean and $90 \%$ confidence intervals (CI) of pharmacokinetic parameters of the test product (Tonizep) compared to the reference product (Aricept).

\begin{tabular}{lccc}
\hline $\begin{array}{l}\text { Pharmacokinetic } \\
\text { parameter }\end{array}$ & Mean & $90 \%$ CI & $\begin{array}{c}\text { Bioequivalence } \\
\text { limit }\end{array}$ \\
\hline Ratio of AUC $_{0-\infty}$ & 1.08 & $1.02-1.14$ & $0.80-1.25$ \\
Ratio of $C_{\max }$ & 1.08 & $0.99-1.17$ & $0.80-1.25$ \\
Difference in $T_{\max }$ & 0.05 & $(-0.19)-0.29$ & \pm 0.41 \\
\hline
\end{tabular}

product based on a randomized, two-way crossover design in fast state with a washout period of 2 weeks. Donepezil concentration in plasma was measured by HPLC with UV detection. The bioequivalence was compared using the parameters: $T_{\max }, \mathrm{AUC}_{0-\infty}$ and $C_{\max }$. The result showed that the $90 \%$ CI of these parameters were within the acceptable range of The Thai FDA guidelines; therefore, this study demonstrated the bioequivalence of the two products.

\section{Conflict of Interests}

All authors do not have a direct financial relation with the commercial identities mentioned in the paper.

\section{Acknowledgments}

This work was supported by T. O. Chemicals (1979) Ltd., Bangkok, Thailand.

\section{References}

[1] D. W. Sifton, Ed., Phyaician's Deak Reference, Medical Economics, Montvale, NJ, USA, 2000.

[2] S. Jackson, R. J. Ham, and D. Wilkinson, "The safety and tolerability of donepezil in patients with Alzheimer's disease," British Journal of Clinical Pharmacology, vol. 58, supplement 1 , pp. 1-8, 2004.

[3] D. Standaert and A. Young, "Treatment of central nervous system degenerative disorder," in Goodman \& Gilman's the Pharmacological Basis of Therapeutics, L. L. Brunton, Ed., pp. 538-540, McGraw-Hill, New York, NY, USA, 11th edition, 2006.

[4] S. L. Rogers and L. T. Friedhoff, "Long-term efficacy and safety of donepezil in the treatment of Alzheimer's disease: an interim analysis of the results of a US multicentre open label extension study," European Neuropsychopharmacology, vol. 8, no. 1, pp. 67-75, 1998.
[5] P. J. Tiseo, K. Foley, and L. T. Friedhoff, "An evaluation of the pharmacokinetics of donepezil $\mathrm{HCl}$ in patients with moderately to severely impaired venal function," British Journal of Clinical Pharmacology, Supplement, vol. 46, supplement 1, pp. 56-60, 1998.

[6] X. Y. Hao, L. Ding, L. M. Li, X. J. Bian, and S. Q. Zhang, "Bioequivalence of donepezil capsule and tablet in human," Yaoxue Xuebao, vol. 38, no. 5, pp. 392-394, 2003.

[7] Y. Lu, H. Wen, W. Li, Y. Chi, and Z. Zhang, "Determination of donepezil hydrochloride (E2020) in plasma by liquid chromatography-mass spectrometry and its application to pharmacokinetic studies in healthy, young, Chinese subjects," Journal of Chromatographic Science, vol. 42, no. 5, pp. 234-237, 2004.

[8] S. L. Rogers and L. T. Friedhoff, "Pharmacokinetic and pharmacodynamic profile of donepezil $\mathrm{HCl}$ following single oral doses," British Journal of Clinical Pharmacology, vol. 46, supplement 1, pp. 1-6, 1998.

[9] A. Ohnishi, M. Mihara, H. Kamakura et al., "Comparison of the pharmacokinetics of E2020, a new compound for Alzheimer's disease, in healthy young and elderly subjects," Journal of Clinical Pharmacology, vol. 33, no. 11, pp. 10861091, 1993.

[10] M. Mihara, A. Ohnishi, Y. Tomono et al., "Pharmacokinetics of E2020, a new compound for Alzheimer's disease, in healthy male volunteers," International Journal of Clinical Pharmacology Therapy and Toxicology, vol. 31, no. 5, pp. 223229, 1993.

[11] R. L. Nation and L. N. Sansom, "Bioequivalence requirements for generic products," Pharmacology and Therapeutics, vol. 62, no. 1-2, pp. 42-55, 1994.

[12] R. Sauter, V. W. Steinijans, E. Diletti, A. Bohm, and H. U. Schulz, "Presentation of results from bioequivalence studies," International Journal of Clinical Pharmacology Therapy and Toxicology, vol. 30, supplement 1, pp. 233-256, 1992.

[13] Criteria and Guideline For the Bioequivalence Study of Generic Drugs, Thai Food and Drug Administration, 2000. 

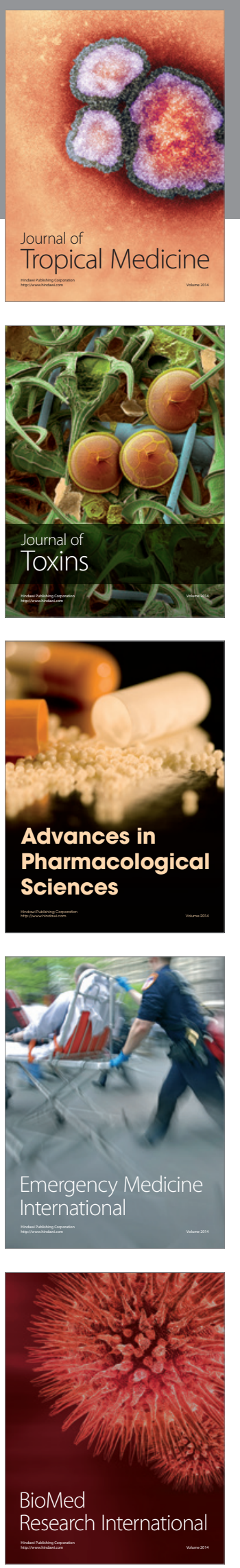
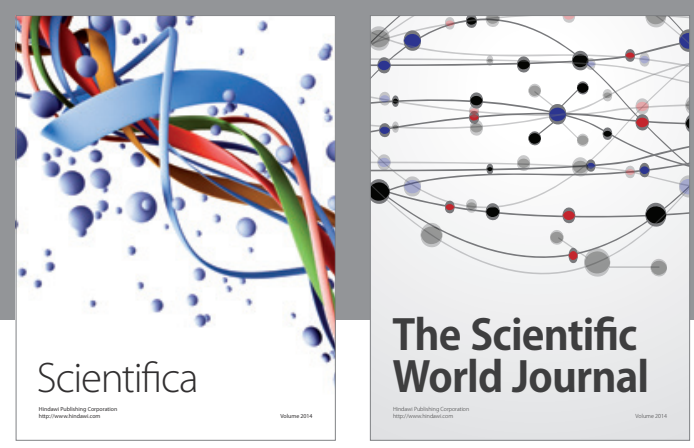

The Scientific World Journal
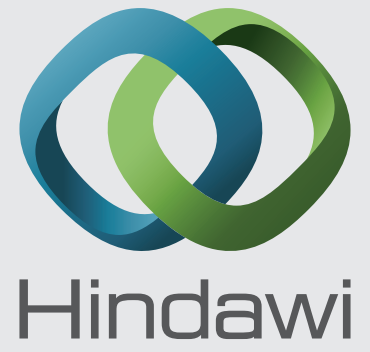

Submit your manuscripts at

http://www.hindawi.com
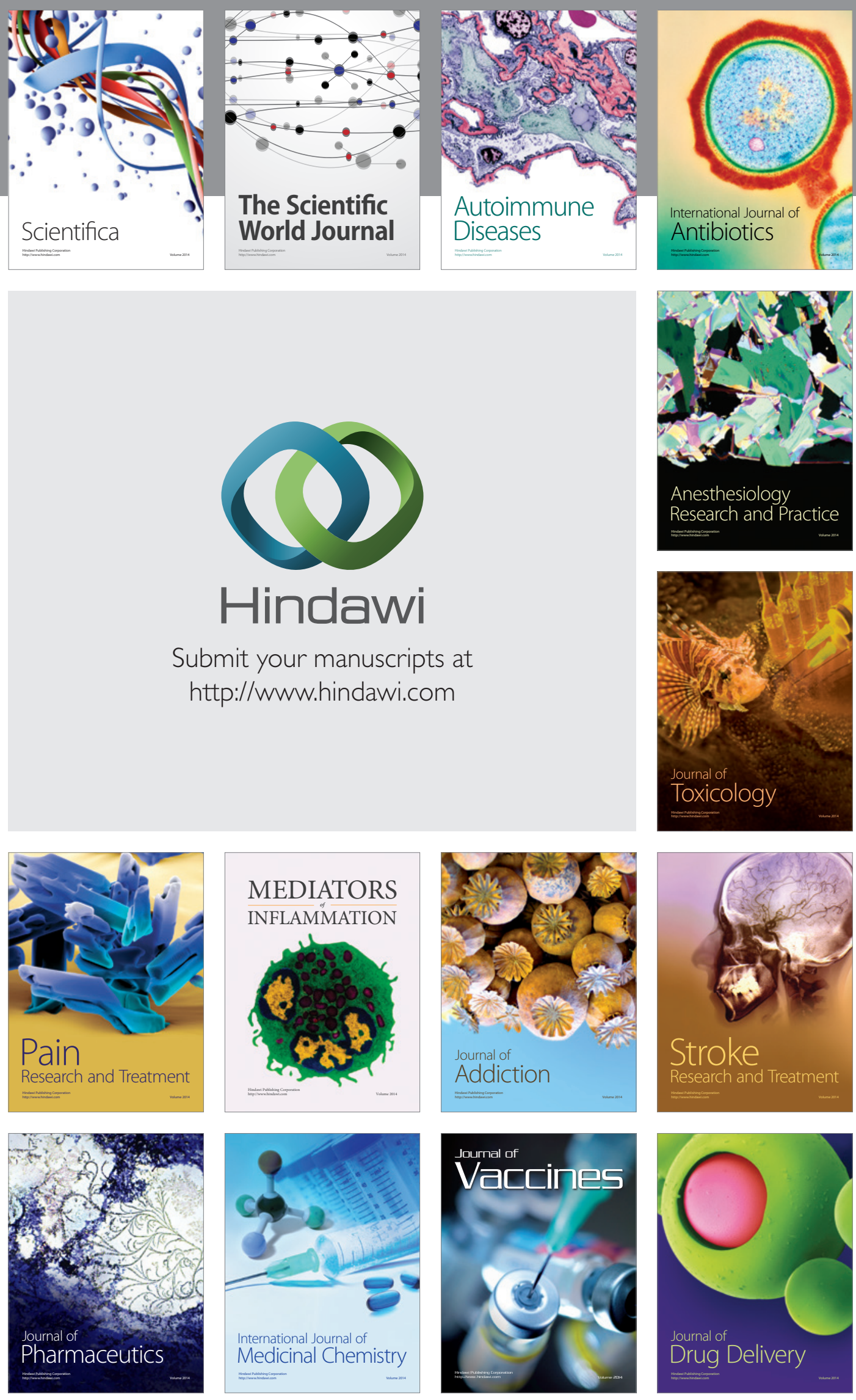